\title{
Erythema exsudativum multiforme induced by a taurine-containing energy drink
}

\author{
Antigona Mithat Begolli Gerqari ${ }^{1}$, Mybera Ferizi² ${ }^{2}$ Sadije Halimi², Aferdita Daka², Syzana Hapciu² ${ }^{2}$ Ilir Mithat Begolli ${ }^{1}$, \\ Mirije Begolli3 ${ }^{3}$ Idriz Hysen Gerqari4
}

\begin{abstract}
Erythema exsudativum multiforme is an immunologically mediated skin condition caused by viruses, bacteria, food, and drugs. There are different forms, and depending on the severity of the disease there is a major and minor form. Whereas the minor form passes without consequences, the major form and Stevens-Johnson syndrome affect the mucosa and may result in death. The disease affects all age groups but is more often observed in young individuals. Typical signs of the disease are skin lesions termed herpes iris. Taurine is an organic compound used in energy drinks and food that can cause many forms of hypersensitivity reactions, and one of these is erythema exsudativum multiforme. As consumption of energy drinks containing taurine increases, the problem of an increase in cases presenting with various forms of hypersensitivity reactions should be considered. Here we present the case of a 19-year-old man with erythema exsudativum multiforme caused by a drink containing taurine. We excluded all other factors that may have caused erythema multiforme and the patient was hospitalized, having been referred to us for the second time presenting with the same problem caused twice by the same drink.
\end{abstract}

Keywords: Erythema exsudativum multiforme, taurine, vasculitis

Received: 24 August 2016| Returned for modification: 22 September 2016| Accepted: 10 October 2016

\section{Introduction}

Erythema multiforme (EM) is an acute (based on hypersensitivity) immune-mediated disease sometimes presenting as a recurrent skin condition (1-10). It is classified as a type IV hypersensitivity immunological reaction. In most cases, erythema exsudativum multiforme is triggered by infections with bacteria, primarily streptococcus, and viruses, especially herpes simplex virus, citomegalovirus, hepatitis virus, HIV, parapoxviruses, and adenoviruses (1-10).

Other factors causing erythema multiforme are drugs and food. The disease affects all age groups; however, it is more often observed in young people. Typical signs of the disease are skin lesions termed herpes iris. The severity of the disease varies and in some cases may also involve the mucosa, in which case one should consider the major form of the disease or Stevens-Johnson syndrome $(2,3,10)$. By consensus definition in 1993, StevensJohnson syndrome was classified separately from the erythema multiforme spectrum and listed under toxic epidermal necrolysis (3).

Taurine is an organic compound, albeit not a free amino acid in the usual biochemical meaning of the term (4). It is naturally found and widely distributed in mammalian tissues (5). In the food industry there are some energy drinks that contain taurine, and there have been reports of hypersensitivity reaction with synthetic taurine (11). Furthermore, taurine has been associated with numerous side effects, and data suggest that taurine can cause reactions such as urticaria, anaphylaxis, and rarely erythema exsudativum multiforme.

\section{Case report}

Here we present the case of 19-year-old student referred to our der- matology clinic for the appearance of sharply demarcated round red macules several centimeters in size (Fig. 1). In some areas of the skin, the macules formed confluent plaques (Fig. 2) localized on both buttocks. In the crural and femoral parts of the skin, target lesions were observed, presenting with a sharp margin round and oval in shape, some of which had a red central blister. Similar lesions, but fewer in number and isolated, were localized on the skin of the abdomen and back. There was no involvement of the mucosa. The patient complained of itching, a local temperature, and discomfort. Corticosteroids and antibiotics were immediately administrated, and topical corticosteroids were applied. Further routine analyses were carried out: sedimentation, hemogram and peripheral blood smear, urea, creatinine, transaminases and bilirubin, glycaemia, and CRP. The values for the parameters measured were within normal range. The TORCH helicobacter pylori and rheumatic factors were also negative. During the medical history, the patient confirmed that this was the second time that he had experienced the same skin changes following consumption of the same taurine-containing energy drink. We carried out a 6-month follow-up of the patient, and there were no recurrences of the disease.

\section{Discussion}

Erythema exsudativum multiforme is an immunologically mediated skin reaction or a reaction to viruses or bacteria (10), classified in the group of type-IV delayed cell-mediated hypersensitivity. The minor form is localized on the skin and the mucosa are not involved $(1,9)$. The typical skin sign is herpes iris, or target lesions with a red to reddish-blue color. The localization of the changes occur in photo-exposed areas. The main causes are various drugs, food containing some additives, bacteria, especially streptococcus, and some viruses, such 


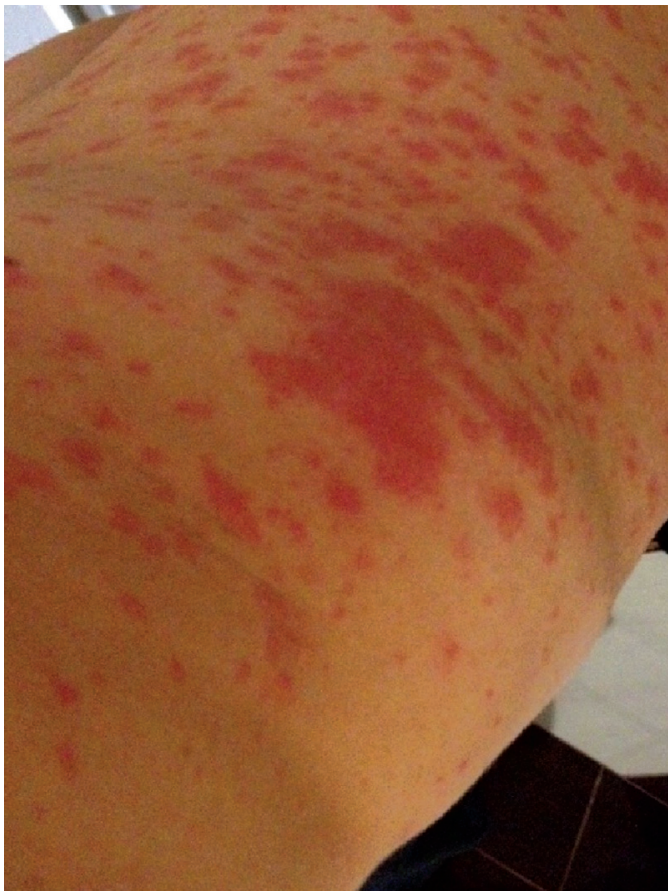

Figure 1 | Erythema exsudativum multiforme.

as herpes simplex virus and Epstein Barr virus in particular. Patients may experience several recurrences per year. Furthermore, the major form of erythema exsudativum multiforme is a rare and life-threatening disease that presents with both skin and mucosal involvement $(2,3,10)$. In such cases, the lesions begin on the face and develop on the trunk with blisters on macular skin lesions (2, 3). Hence, the same disease appears at different stages and with different severity. Although Stevens-Johnson syndrome mostly appears as a reaction to some medicines, it has been separated from the erythema multiform spectrum and added to toxic epidermal necrolysis (3). Mucosal involvement in a situation in which erythema multiforme is caused by herpes simplex must be taken into consideration when differentiating between erythema mul-

\section{References}

1. Sokumbi 0, Wetter DA. Clinical features, diagnosis, and treatment of erythema multiforme: a review for the practicing dermatologist. Int J Dermatol. 2012;51: 889-902.

2. Assier H, Bastuji-Garin S, Revuz J, Roujeau JC. Erythema multiforme with mucous membrane involvement and Stevens-Johnson syndrome are clinically different disorders with distinct causes. Arch Dermatol. 1995;131:539-43.

3. Bastuji-Garin S, Rzany B, Stern RS, Shear NH, Naldi L, Roujeau JC. Clinical classification of cases of toxic epidermal necrolysis, Stevens-Johnson syndrome, and erythema multiforme. Arch Dermatol. 1993;129:92-6.

4. Lombardini JB, Schaffer SW. Special issue: Taurine: discovered 185 years ago and still intrigues the scientific community. Amino Acids. 2002;23:343.

5. Lambert IH. Regulation of the cellular content of the organic osmolyte taurine in mammalian cells. Neurochem Res. 2004;29:27-63.

6. Choi MJ, Kim JH, Chang KJ. The effect of dietary taurine supplementation on plasma and liver lipid concentrations and free amino acid concentrations in rats fed a high-cholesterol diet. Adv Exp Med Biol. 2006;583:235-42.

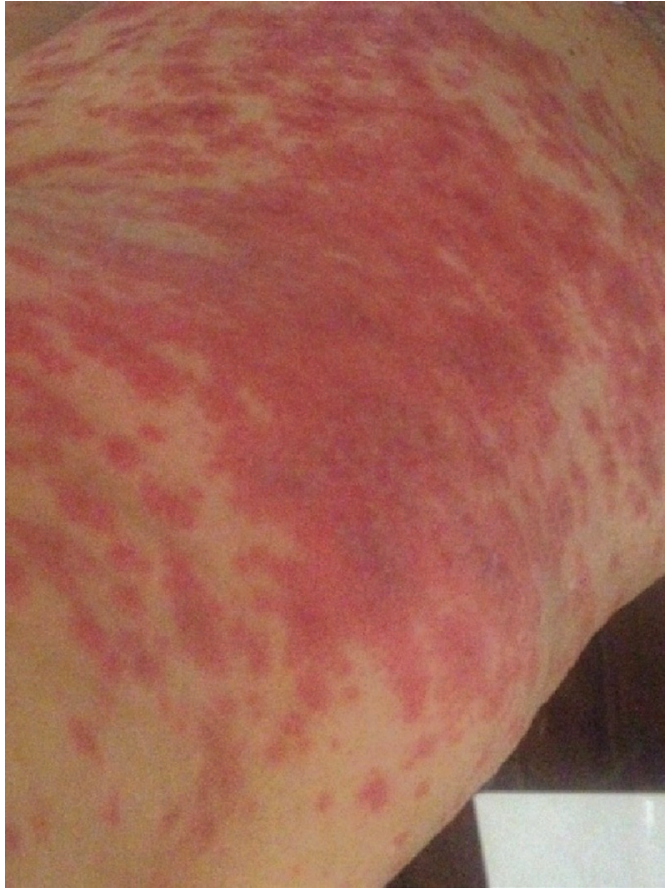

Figure 2 | Erythema exsudativum multiforme: confluent plaques.

tiforme and Stevens-Johnson syndrome $(2,3,10)$. The prognosis of the disease varies and depends on the cause and the state of the patient's immune system. Energy drinks containing taurine have recently been blamed for causing hypersensitivity reactions such as urticaria, in some cases anaphylaxis, and in rare cases even erythema exsudativum multiforme (8). Taurine, an organic compound found in animal tissues (5), has been studied in the medical and pharmaceutical industry as a food and drink supplement that lowers the risk of cardiovascular disease, mostly via a mechanism that prevents hypertension and decreases blood cholesterol (6). Nevertheless, it is important to emphasize the potential hypersensitivity reaction to synthetic taurine (11).

7. Huston RK, Baxter LM, Larrabee PB. Neonatal parenteral nutrition hypersensitivity: a case report implicating bisulfite sensitivity in a newborn infant. JPEN J Parenter Enteral Nutr. 2009;33:691-3.

8. Meng WJ, Li Y, Zhou ZG. Anaphylactic shock and lethal anaphylaxis caused by compound amino acid solution, a nutritional treatment widely used in China. Amino Acids. 2012;42:2501-5.

9. Huff JC. Erythema multiforme and latent herpes simplex infection. Semin Dermatol. 1992;11:207-10.

10. Fitzpatrick TB et al., editors. Fitzpatrick's dermatology in general medicine. 4 th ed. New York (USA): McGraw-Hill; c1993. Fritsh PO, Elias PM. Erythema multiforme and toxic epidermal necrolysis; p. 585-60o.

11. Lee SE, Lee SY, Jo EJ, Kim MY, Yang MS, Chang YS, et al. A case of taurine-containing drink induced anaphylaxis. Asia Pac Allergy. 2013;3:70-3. 